\title{
Repensando los indicadores educativos: la gestión educativa, curricular y de vinculación con la comunidad
}

\author{
María del Rocío Ramírez-González* \\ Jorge Quesada-Lacayo** \\ Investigadora del Área de Investigación y Calidad Educativa del Colegio de Licenciados \\ y Profesores en Letras, Filosofía, Ciencias y Artes de Costa Rica (COLYPRO), Costa Rica \\ rramirez@colypro.com \\ Investigador de la Unidad de Investigación Laboral del Colegio de Licenciados \\ y Profesores en Letras, Filosofía, Ciencias y Artes de Costa Rica (COLYPRO), Costa Rica \\ jquesada@colypro.com
}

Recibido: 09 de agosto del 2018

Corregido: 03 de octubre del 2018

Aceptado: 27 de marzo del 2019

\begin{abstract}
Resumen
El artículo se centra en una revisión de literatura, mediante la cual se sistematizan los principales indicadores de gestión educativa tematizados por la bibliografía revisada, con el objetivo de identificar cuáles indicadores de gestión administrativa, curricular y de vinculación con la comunidad, a nivel nacional e internacional, son utilizados para explicar el desempeño educativo y el éxito escolar. Los hallazgos de esta primera etapa de investigación plantean la necesidad de pensar la gestión de los procesos educativos desde la diversidad y especificidad de su contexto local e institucional, así como el democratizar los espacios educativos, en la medida en que se incorpora a nuestros procesos de gestión el ámbito comunitario, ambas dimensiones nutren el currículo escolar, su comprensión y las prácticas que propician aprendizajes exitosos; aspectos que cobran importancia en el marco de las transformaciones curriculares en curso en nuestro sistema educativo.
\end{abstract}

Palabras clave: Gestión educativa; política educativa; indicadores educativos; comunidad educativa; currículo educativo.

\section{Abstract \\ Rethinking educational indicators: The educational management, curricular and of connection with the community}

This article focuses on a review of literature, through which the leading indicators of educational management, schematized by the revised bibliography, with the objective of identifying which indicators of administrative management, curricular management and its relation with the community at national and international scenarios, are used to explain educational performance and school success. The findings of this first stage, raised the need to think about the management of educational processes from the diversity and specificity of their local and institutional context. Likewise, as well as democratize educational spaces to the extent that incorporates at our educational processes in the community. Both dimensions enrich the school curriculum, its understanding and the practices that promote successful learning

Key words: Education Management; educational policy; educational indicators; educative community; educational curriculum. 


\section{INTRODUCCIÓN}

En los últimos años, entidades nacionales como el Programa Estado de la Nación a través de sus informes del Estado de la Educación, así como de las agencias internacionales como la Organización para la Cooperación y el Desarrollo Económicos (OCDE) han realizado esfuerzos por investigar y analizar los desafíos educativos y determinar aquellos factores clave que permitan superarlos, con el objetivo de mejorar el rendimiento escolar, promover la justicia y la inclusión social e incidir en el desarrollo del país.

Los informes nacionales e internacionales de seguimiento al desempeño del sistema educativo han buscado relacionar los múltiples factores que inciden en el logro de los aprendizajes. Algunas variables que se han considerado son aquellas vinculadas con: a) los estudiantes y sus familias; b) los docentes y las prácticas de clase; c) las características de los centros educativos y su gestión.

Asimismo, estos estudios han demostrado que los contextos institucionales influyen de manera significativa en la práctica docente que, a su vez, muestra efectos directos en los logros educativos de las personas estudiantes, (OCDE, 2009, 2014, Leithwood 2009, Cornejo, Rubilar, Díaz \& Rubilar, 2014), así se han desarrollado distintos indicadores educativos cuya finalidad es movilizar y orientar recursos en aquellas áreas que requieren mayor compromiso institucional.

La idea subyacente es que las nuevas formas de gestionar lo público vayan de la mano con una toma de decisiones fundamental, para ello la evaluación educativa adquiere una nueva centralidad en la discusión de la agenda pública, no solo para valorar el grado de calidad de los sistemas educativos, sino además el interés de diversos grupos sociales porque se alcancen las metas educativas planteadas por la agenda nacional en el marco de lo que se espera internacionalmente en términos de eficiencia y eficacia (Meza, 2008).

La mayoría de los indicadores utilizados para ese fin enfatizan en las evaluaciones estandarizadas sobre el desempeño de los aprendizajes y sus factores asociados, utilizando indicadores que operan en los niveles macro, lo que plantea retos para establecer una lectura más centrada en las dinámicas institucionales (Martin \& Martínez, 2009). Por otro lado, a partir de la revisión bibliográfica se determina que son pocos los esfuerzos realizados en el ámbito nacional con miras a profundizar la dinámica e interacciones existentes entre las dimensiones: administrativa, curricular y de vinculación con la comunidad en contextos educativos específicos.

En relación con lo expuesto, y considerando lo fundamental de estas dimensiones en la gestión educativa y su impacto significativo en el rendimiento escolar, se concibe la investigación titulada Construcción de indicadores nacionales de gestión administrativa y curricular de centros educativos para el mejoramiento del desempeño educativo, un esfuerzo en conjunto entre la carrera Administración Educativa de la Universidad Estatal a Distancia y el Colegio de Licenciados y Profesores en Letras, Filosofía, Ciencias y Artes, con base en la que hemos buscado identificar cuáles indicadores de gestión administrativa, curricular y de vinculación con la comunidad a nivel nacional e internacional son utilizados para explicar el desempeño educativo y el éxito escolar.

A su vez, pretendemos coordinar esfuerzos para la construcción de indicadores en gestión administrativa y curricular de centros educativos, y de vinculación con la comunidad, procurando simultáneamente avanzar en la implementación de políticas educativas que permitan mejorar las condiciones institucionales donde ocurren las prácticas de aula y por tanto inciden en el rendimiento escolar, desde el fortalecimiento de la formación de gestores educativos.

El país requiere avanzar en la construcción de indicadores de gestión para centros educativos que permitan el seguimiento de variables relacionadas con ambientes de aprendizaje que propician el éxito educativo, desde la perspectiva de inclusión y justicia social; además, indicadores que guíen la toma de decisiones y que permitan rendir cuentas del progreso y los cambios en el centro educativo. 
A continuación, se presentan los principales hallazgos de la primera etapa de investigación, en la cual se realizó una revisión bibliográfica y sistematización de las mimas con base en las dimensiones de análisis propuestas. En el escrito se aborda inicialmente el papel de la gestión educativa en la política estatal. Posteriormente, se desarrollan los indicadores en su nivel curricular, administrativo y de vinculación con la comunidad, para finalmente esbozar un balance general.

\section{METODOLOGÍA}

El estudio de investigación se centró en tres dimensiones, la gestión curricular, la administrativa y la de vinculación con la comunidad, la búsqueda se realizó considerando fuentes secundarias, tales son libros, revistas de divulgación o de investigación científica indexadas y arbitradas, sitios web, a nivel nacional e internacional.

Para la gestión de apoyo documental se utilizó el software Zotero, el cual se basa en la organización por categorías de la documentación revisada y su referenciación por formato; así mismo se utilizó una hoja de cálculo para la organización de la información con base en las tres dimensiones propuestas.

Entre las bases de datos consultadas a nivel internacional se encuentran: la Organización de las Naciones Unidas para la Educación, la Ciencia y la Cultura - UNESCO, la Organización de Estados Iberoamericanos - OEl, y la Organización para la Cooperación y el Desarrollo Económicos - OCDE; a nivel nacional se revisaron las revistas de educación de las universidades estatales de Costa Rica, entre las palabras claves están: gestión educativa, gestión administrativa, gestión del currículo, gestión curricular, vínculos comunidad - centro educativo, gestión educativa y comunitaria, para ello la revisión por internet se visualizó a través de operadores lógicos, los cuales incluyeron los términos específicos por investigar.

El papel de la gestión educativa en la política educativa

En nuestro país la política de Estado en materia de educación la dirige el Consejo Superior de Educación - CSE, instancia que posee rango constitucional para dictar los lineamientos y orientaciones generales del sistema educativo costarricense.

A través de las distintas políticas emitidas por el ente citado, la gestión del centro educativo se ha constituido como un elemento fundamental dentro de los procesos de enseñanza y aprendizaje. En el año 2008, fue aprobada la política de estado el Centro Educativo de Calidad como Eje de la Educación Costarricense y recientemente en el año 2017 entró a regir la política educativa La persona: centro del proceso educativo y sujeto transformador de la sociedad, así se realizó un viraje importante en el foco de atención, colocando el énfasis en el sujeto de aprendizaje y en los procesos necesarios para llevar a cabo la labor educativa.

En la primera, el centro educativo viene a recobrar el reconocimiento de instancia mediadora, sobre la cual, y partiendo de sus potencialidades y posibles puntos de mejora, es posible hablar de una calidad de la educación con carácter universal:

[...] al centro educativo de calidad la condición de eje central de la política educativa nacional y pilar esencial de la operación del sistema, es la idea-fuerza que propiciará la construcción de centros educativos con una identidad institucional más rica, con mayor autonomía relativa, capacidad de decisión y con una participación más amplia de la comunidad educativa, constituida por estudiantes, padres de familia, personal del centro educativo y miembros de la comunidad local. (CSE, política de estado el Centro Educativo de Calidad como Eje de la Educación Costarricense, pp. 6-7) 
De esta manera, la política educativa respondía a la necesidad de brindarle mayor autonomía a los centros educativos, en un contexto en el cual se planteaban discusiones en torno a descentralizar procesos y desarrollar capacidades organizacionales que permitieran responder a las necesidades de su contexto local en un marco institucional en donde opera un sistema educativo centralizado.

Si bien esta política educativa fue aprobada en el año 2008, la puesta en práctica y ejecución aún no ha permeado los distintos niveles y espacios de toma de decisiones, ni mucho menos a los diferentes actores de la comunidad educativa, asentada en una cultura burocrática y centralista, que impide la puesta en práctica de una gestión escolar que se adapte al contexto y a las necesidades de nuestro tiempo (Cortés, 2016).

La entrada en vigencia de una nueva política educativa a finales del año 2017, involucra y plantea nuevos retos en el marco de las transformaciones curriculares en curso, las nuevas demandas sociales hacia una educación inclusiva y con justicia social y en las diferentes articulaciones entre los procesos de enseñanza y aprendizaje y su entorno, local y global, lo que requiere de análisis y profundización sobre el impacto de la política en la gestión escolar.

Por tanto, se necesita que a partir de este nuevo modelo de referencia educativo nacional se cuente con indicadores de gestión que coadyuven a la materialización de esta política y al monitoreo de su impacto, considerando siempre las realidades tan complejas y diferentes de los centros educativos.

\section{HACIA UN ESTADO DE LA CUESTIÓN EN INDICADORES DE GESTIÓN EDUCATIVA}

Para una mejor comprensión del sistema educativo costarricense, se hace necesaria la construcción de indicadores que permitan una representación más completa de la realidad socioeducativa. Avanzar hacia la delimitación de indicadores es contar con información para la toma de decisiones.

Un ámbito clave es la gestión, como elemento que brinda coherencia al conjunto de las actividades educativas. Al respecto Casassus (1999) señala que la gestión se relaciona con los componentes de una organización en cuanto a sus arreglos institucionales, la articulación de recursos, los objetivos y, por, sobre todo, con las interrelaciones entre las personas en la acción.

Según Antúnez (1998), la palabra gestión implica describir o analizar el funcionamiento de la institución escolar. En consecuencia, es sinónimo de integración con la realidad material, cultural, organizativa y pedagógica. La gestión constituye articulación entre el ámbito educativo y los diferentes sectores sociales.

La gestión educativa constituye el conjunto de actuaciones que se desarrollan en el centro como expresión del gobierno institucional, bajo la conducción y responsabilidad del director, para definir sus objetivos y diseñar la naturaleza y desarrollo de las tareas para alcanzarlos. Implica la participación de todos los miembros en la planificación, ejecución y control de las decisiones y acciones que se toman en los ámbitos: curricular, servicios, recursos y comunidad, en grado y forma acorde con sus capacidades, competencias y roles (Bellido, Díaz, Morales \& Pino, 2009).

\section{GESTIONANDO EL CURRÍCULO EN CENTROS EDUCATIVOS}

El punto de partida para entender la dimensión, lo constituye el currículo escolar. De acuerdo con Poggi (1998) es la forma en la que una sociedad se enfrenta al problema de organizar un conjunto de prácticas educativas con diversas teorías y técnicas. La gestión curricular resulta medular en las acciones que 
lleva a cabo el gestor educativo y los actores institucionales responsables de hacer efectivo el currículo. Podría afirmarse que las dimensiones administrativa y comunitaria nutren a la parte curricular que sustenta las actividades y la organización de todo espacio socioeducativo.

La revisión bibliográfica nos permitió desarrollar al menos tres subdimensiones, la primera sobre los aspectos del currículo, su planificación y evaluación; la segunda vinculada con lo material, la cual se refiere a los indicadores que nos permiten comprender con cuáles recursos contamos para la implementación del currículo; finalmente, la cultural que nos permite una lectura del contexto tanto institucional como local.

TABLA 1

Indicadores de gestión curricular

\begin{tabular}{lll}
\multicolumn{1}{c}{ Subdimensiones } & \multicolumn{1}{c}{ Indicador } & \multicolumn{1}{c}{ Aspecto de medición } \\
Curricular & Planificación & Cumplimiento de los programas educativos \\
& & Conocimiento del plan de estudios \\
& & Estrategias de programación del proceso educativo \\
& Metas y objetivos institucionales \\
& Desarrollo del plan anual de trabajo \\
& Formación de equipos \\
\cline { 2 - 3 } & Evaluación & Orientaciones institucionales \\
& Supervisión y seguimiento docente \\
& Articulación del equipo curricular \\
& Prácticas evaluativas \\
& Acompañamiento a la población estudiantil \\
& Manejo de resultados \\
\hline Material & Espacios de estudio \\
& Bibliotecas \\
& Tecnología para los procesos de aprendizaje \\
& Organización de la infraestructura \\
\hline Cultura & Principios institucionales \\
& Procedimientos para el personal y la población estudiantil \\
& Participación en los espacios del centro \\
& Medios de comunicación y reunión de los actores educativos \\
& Prestación de los servicios \\
& Liderazgo del director \\
& Seguimiento de las acciones docentes \\
\hline Formación del recurso humano & Capacitaciones \\
& Equipos de formación continua \\
& Competencias profesionales \\
\hline
\end{tabular}

Fuente: Elaboración propia del equipo investigador.

Los artículos y la documentación revisada, tanto a nivel internacional como nacional señalan consistentemente la necesidad de monitorear y evaluar las variables relacionadas con la gestión curricular, con el fin de realizar las transformaciones necesarias y así lograr resultados más alentadores en torno al rendimiento escolar de los y las estudiantes. 
Los aspectos de medición incorporados en ésta dimensión involucran un fuerte acompañamiento a la labor docente, así como a los equipos y procesos de apoyo en el ejercicio profesional, lo que pone en el centro de la discusión aquellos elementos que tiene un impacto real en la práctica de aula.

\section{GESTIONAR LA COMUNIDAD EDUCATIVA: LOS VÍNCULOS EXTERNOS Y ADMINISTRACIÓN ESCOLAR}

En el apartado realizaremos una descripción de las principales tendencias e indicadores en las formas de articulación entre el centro escolar y la comunidad, el análisis se focaliza en pensar las vinculaciones bajo la perspectiva de las comunidades de aprendizaje (Aiguadé, Saso, Gallart \& Carol, 2003, Díez-Palomar \& Flecha García, 2010, Mingorance \& Estebaranz, 2009), cuyo objetivo es crear aprendizajes significativos en el marco de una participación activa de los múltiples actores que conforman la comunidad.

La literatura ha identificado al menos tres modelos para pensar los vínculos entre los centros educativos y los proyectos de comunidad o su entorno comunitario. El primero de ellos piensa a la escuela y a su cultura como un elemento externo a la comunidad (González, 2004). Esta idea es la continuación del origen mismo de los sistemas educativos, los cuales se articularon a partir de una lógica vertical, pues se constituyeron en una especie de fortaleza especializada donde se concentraban el saber científico y la formación en valores necesarios para los nacientes estados nacionales, en el contexto del proyecto de modernización capitalista, ésta concepción privilegió un adentro y un afuera de los centros educativos, una línea divisoria entre la comunidad y la escuela (Tenti, 2004).

En el segundo modelo, lo comunitario y lo educativo se diluyen en un solo espacio, impactando en los procesos educativos y perdiendo la especificidad necesaria para avanzar en el currículo (González, 2004).

En este modelo la creación de vínculos entre el centro educativo y su comunidad se realiza desde un espacio institucionalizante, se favorece la reproducción de formas verticales y burocráticas de participación comunitaria (Tenti, 2004), y se incorporan una serie de condicionantes sociales, políticos y culturales que impiden procesos de aprendizaje significativos.

Un tercer modelo se refiere a la construcción de vínculos a partir de la articulación de los actores educativos y comunitarios. Se fundamenta en la creación de un proyecto común, donde los diferentes actores se apropian del proceso educativo y trabajan para lograr objetivos colectivos. En esta modalidad la escuela es el centro de mediación para lograr procesos de aprendizaje de sus estudiantes, al respecto Neirotti y Poggy (1998, citado por González 2004) señalan:

El sistema educativo formal no monopoliza el proceso de aprendizaje, ni son los docentes los únicos que enseñan. Las organizaciones comunitarias y los espacios públicos locales son ámbitos donde los sujetos aprenden, incorporando valores y hábitos, desarrollando capacidades (sean éstas para producir bienes o servicios o para ejercer sus derechos y responsabilidades ciudadanas). Las comunidades, a su vez, cuentan con sus propias instituciones y agentes de enseñanza y aprendizaje: familias, iglesias, clubes, organizaciones de vecinos, bibliotecas, mercados, fábricas, organizaciones productivas. En consecuencia, resulta necesario observar qué se aprende en esta comunidad donde la escuela es un actor más y quiénes son los actores de este proceso de aprendizaje. (p.15)

A partir de esta discusión, rescatamos dos indicadores valiosos que nos brindan una idea para construir las Ilamadas comunidades de aprendizaje, los cuales se resumen en la tabla 2: 
TABLA 2

Indicadores de gestión comunitaria

Indicador

Acciones educativas comunitarias

Acciones referidas a la inclusión escolar
Aspecto de medición

Acciones cuyo objetivo es generar espacios de comunicación e intercambio tendiente a mejorar las condiciones educativas de los niños y los jóvenes, y construir una retroalimentación cultural mutua.

Fuente: González (2004).

\section{LA GESTIÓN ADMINISTRATIVA: ELEMENTOS CENTRALES}

Los centros educativos son pieza fundamental dentro de las dinámicas socioeducativas, las cuales se enmarcan dentro de aspectos relacionados con los procesos de aprendizaje, hasta la constitución de componentes culturales y sociales, representados por medio de las habilidades sociales y las creencias adquiridas. El centro educativo se torna, por lo tanto, en uno de los puntos físicos más importantes en la vida de las personas, y de las comunidades enteras. Rodríguez (2016), detalla que el entorno de aprendizaje se presenta gracias a la capacidad administrativa que permite la gestión educativa y que engloba aspectos administrativos, actores que forman parte de la institución y a su vez se relacionan coherentemente con el currículo escolar.

La UNESCO (2016), por ejemplo, ha planteado una serie de recomendaciones que centran su mirada hacia la gestión de los centros educativos, en el documento Educación 2030. Declaración de Incheon y marco de acción para la realización del objetivo de desarrollo sostenible 4 se establecen dos recomendaciones:

- Reforzar la eficacia y eficiencia de las instituciones, la administración escolar y la gobernanza ampliando la participación de las comunidades, incluidos los jóvenes y los padres, en la gestión de las escuelas.

- (Los procesos participativos) deben comenzar con el compromiso de las familias y comunidades de reforzar la transparencia y garantizar una buena gestión de la administración de la educación. (p. 37)

Por lo tanto, podemos entender la dimensión administrativa como aquella en la que se desarrollan gestiones desde el centro educativo, precisamente desde la administración del centro educativo, tomando en consideración estrategias que involucren a la comunidad, a la población docente y estudiantil, asimismo fortalezcan las dinámicas curriculares y profesionales con miras al mejoramiento de las capacidades comunales de manera integral.

Tomando en consideración el enfoque descrito, la dimensión administrativa debe trascender los aspectos plenamente tradicionales mantenidos dentro del centro educativo, y lograr una gestión que sobrepase los límites y proyecte capacidades de involucramiento que alcancen a la población docente, estudiantil y sus familiares, por tanto, a la comunidad. 
Diversos autores (Álvarez \& Martínez, 2016, Cerdas, Torres, \& García, 2016) visualizan los centros educativos como ejes motivadores de mejoras comunales, a partir de una gestión administrativa que supere los límites físicos del propio centro. Obsérvese el cuadro comparativo de las propuestas consideradas en la presente investigación:

TABLA 3

Componentes de la dimensión administrativa

\begin{tabular}{|c|c|}
\hline Subdimensiones & Aspectos de medición \\
\hline \multicolumn{2}{|l|}{ Álvarez \& Martínez, 2016} \\
\hline Familia & $\begin{array}{l}\text { Relación de la familia con el centro educativo y el proceso de formación de la persona } \\
\text { estudiante. }\end{array}$ \\
\hline Docentes & $\begin{array}{l}\text { Rol de la persona docente dentro del proceso de formación de la persona estudiante, a partir } \\
\text { de las condiciones del centro educativo. }\end{array}$ \\
\hline Centro escolar & Espacio físico donde se genera el proceso educativo y las relaciones docente-estudiante. \\
\hline Sistema educativo y social & $\begin{array}{l}\text { Proceso en el que se relacionan los procesos educativos que articulan las situaciones sociales o } \\
\text { de contexto. }\end{array}$ \\
\hline Estudiantes & Población que se está formando dentro del proceso educativo. \\
\hline \multicolumn{2}{|l|}{ Cerdas, Torres \& García, 2016} \\
\hline Organismos de centro & Diversas instancias del centro educativo. \\
\hline Redes de apoyo & $\begin{array}{l}\text { Relaciones que existen entre las familias con los grupos de estudiantes, así como el apoyo de la } \\
\text { población docente en los procesos educativos. }\end{array}$ \\
\hline Gestión talento humano & $\begin{array}{l}\text { Manejo desde el centro educativo del personal docente y sus labores profesionales y de } \\
\text { capacitación. }\end{array}$ \\
\hline Infraestructura & el centro educativo que influyen en el proceso de formación. \\
\hline
\end{tabular}

Fuente: Elaboración propia a partir de la propuesta de Álvarez \& Martínez (2016), Cerdas, Torres \& García (2016).

Con base en el cuadro comparativo, se logra notar que los elementos propuestos por ambos autores reflejan la búsqueda de involucrar a la comunidad en el proceso de gestión del centro educativo. De esta forma, la propuesta para los componentes de esta dimensión correspondientes a la construcción de indicadores, sería la siguiente: 
TABLA 4

Indicadores de la gestión administrativa

\begin{tabular}{|c|c|}
\hline Subdimensión & Aspectos de medición \\
\hline Gestión del centro educativo & $\begin{array}{l}\text { Todo lo relacionado con temas de infraestructura y elementos físicos, como } \\
\text { ambientes sin contaminantes y adecuados para la población estudiantil y docente. }\end{array}$ \\
\hline Gestión articuladora con la comunidad & $\begin{array}{l}\text { Entendida como aquellas acciones y estrategias desarrolladas para el } \\
\text { involucramiento de las familias y la comunidad. }\end{array}$ \\
\hline Gestión del talento humano docente & $\begin{array}{l}\text { Todas aquellas acciones que fortalezcan el quehacer docente, por medio de } \\
\text { condiciones óptimas que permitan la proyección de motivaciones y la comprensión } \\
\text { del contexto de los estudiantes. Sería uno de los agentes de cambio más relevantes. }\end{array}$ \\
\hline Gestión familiar y redes de apoyo & $\begin{array}{l}\text { La capacidad, por parte del centro educativo, de articular a las familias, con el fin } \\
\text { de mejorar las condiciones óptimas para los estudios, así como las estrategias } \\
\text { para que sean parte de la toma de decisiones y construcción de acciones para el } \\
\text { fortalecimiento de las capacidades de acción educativas. }\end{array}$ \\
\hline
\end{tabular}

Fuente: Elaboración propia.

\section{CONCLUSIONES}

La demanda por una educación con justicia social, inclusiva, equitativa, democrática y de calidad, permeada por la perspectiva de derechos humanos implica nuevas formas de gestionar la educación y de reconocer los elementos necesarios para enfrentar los retos que la sociedad le exige.

Para encarar los desafíos se requiere estimular aquellos factores que permiten el logro de los aprendizajes de las poblaciones estudiantiles, y movilizar los diferentes recursos y procesos hacia las dimensiones que necesitan mayor compromiso institucional. De esta forma, se visualiza el uso de indicadores de gestión educativa como una herramienta que propiciará cambios y logros sustanciales, respetando y reconociendo la especificidad de las instituciones educativas.

La literatura revisada converge en elementos que se deben considerar desde el campo de la administración educativa. La primera de ellas es la necesidad de pensar la gestión de los procesos educativos desde la diversidad y especificidad de su contexto local e institucional, en esa línea de trabajo, los indicadores relacionados con la dimensión administrativa, brindan elementos contextuales que pueden alimentar la práctica de aula y el desarrollo de proyectos colaborativos entre los docentes y los actores educativos.

Un segundo aporte hace referencia a la necesidad de democratizar los espacios educativos en la medida en que incorporamos a nuestros procesos de gestión el ámbito comunitario, su contexto y la participación de madres y padres dentro de las responsabilidades del éxito escolar, en esta dimensión encontramos indicadores en dos líneas, la primera en referencia a aquellas acciones que buscan mejorar las condiciones educativas de los niños / jóvenes, y las acciones referidas a la inclusión escolar, el cual envuelve un abordaje participativo con diversos actores de la comunidad.

Ambas dimensiones nutren el currículo escolar, su comprensión y las prácticas que propician aprendizajes exitosos, lo cual tiene implicaciones, no solo en la gestión de los centros educativos, sino también en el ejercicio de la docencia y las prácticas de aula. Lo anterior cobra importancia en el marco de las transformaciones curriculares experimentadas en el sistema educativo costarricense, aspecto que ha sido explicitado en la nueva política educativa, aprobada recientemente por el Consejo Superior de Educación. 
A partir de estos primeros hallazgos, se fortalece el debate sobre la necesidad de repensar la gestión de centros educativos desde un paradigma que combine el uso de información para la toma de decisiones en un marco de inclusión y justicia social, lo cual no solo trasciende el ámbito técnico del uso de indicadores, sino que plantea y confronta nuestras prácticas como gestores educativos.

\section{REFERENCIAS}

Aiguadé, I. P., Saso, C. E., Gallart, M. S. \& Carol, M. R. V. (2003). Comunidades de aprendizaje: transformar la educación (Vol. 177). Graó. Retrieved from https://books.google.com/books?hl=en\&lr=\&i$\mathrm{d}=0 Y 5 \mathrm{lb} \_J v e 4 \mid \mathrm{C} \& \mathrm{i}=$ fnd\&pg $=$ PA6\&dq=\%22edici\%C3\%B3n:+noviembre\%22+\%22de+la+misma+ por+cualquiera+de+sus+medios+tanto+si+es+el\%C3\%A9ctrico,+como+qu\%C3\%ADmico,+mec\%C3\%A1nico,+\%C3\%B3ptico,\%22+\%22social+y+diversidad+de+opciones+de+vida$+\% 27 \% 22+\& o t s=H 5$-iMjQpP5\&sig=J2dAlwCAzM2-2-obmk6ZaSZI45g

Álvarez, L. \& Martínez-González, R.-A. (2016). Cooperación entre las familias y los centros escolares como medida preventiva del fracaso y del riesgo de abandono escolar en adolescentes. Revista latinoamericana de educación inclusiva, 10(1), 175-192.

Antúnez, S. (1998). Claves para la organización de centros escolares (IV). Horsori. Recuperado a partir de http://www.terras.edu.ar/aula/cursos/8/biblio/8ANTUNEZ-Serafin-CAP8-Innovacion-y-Cambio-en-los-Centros-Escolares.pdf

Bellido, A. C., Díaz Donado, J., F. Morales Ríos \& Pino de Ochoa, L. (2009). Gestión y Supervisión en el Centro de Educación Básica (Vol. 36). San José Costa Rica: Coordinación Educativa y Cultural Centroamericana. Recuperado a partir de http://www.ceducar.info/ceducar/recursos/biblioteca-virtual/volumen36.pdf

Casassus, J. (1999). Marcos conceptuales para el análisis de los cambios en la gestión de los sistemas educativos. En La gestión: en busca del sujeto. Santiago de Chile. UNESCO. Recuperado a partir de http://unesdoc.unesco.org/images/0011/001176/117612so.pdf

Cerdas Montano, V., Torres Vitoria, N. \& García Martínez, J. A. (2016). Tendencias y desafíos de la administración educativa costarricense: La génesis de un proyecto. Gestión de la educación, 6(2), 119. https://doi.org/10.15517/rge.v1i2.25495

Consejo Superior de Educación. (2008). El Centro Educativo de calidad como Eje de la Educación. San José Costa Rica.

Consejo Superior de Educación. (2017). La persona: centro del proceso educativo y sujeto transformador de la sociedad. San José Costa Rica.

Cornejo, C. O., Rubilar, F. C., Díaz, M. C. \& Rubilar, J. C. (2014). Cultura y liderazgo escolar: factores claves para el desarrollo de la inclusión educativa/Culture and school leadership: key factors to develop an inclusive education. Revista Actualidades Investigativas en Educación, 14(3). Recuperado a partir de http://revista.inie.ucr.ac.cr/index.php/aie/article/view/636

Cortés Rodríguez, B. (2016). La gestión anacrónica de los centros educativos en Costa Rica. Gestión de la educación, 6(2), 137. https://doi.org/10.15517/rge.v1i2.25498

Díez-Palomar, J. \& Flecha García, R. (2010). Comunidades de Aprendizaje: Un proyecto de transformación social y educativa. Revista Interuniversitaria de Formación Del Profesorado, 24(1). Retrieved from http://www.redalyc.org/html/274/27419180002/

González, T. D. (2004). "Política Del Ministerio de Educación Y Ciencia En Temas de Educación de Adultos (1970-1990)." Pp. 81-112 in Política de educación de adultos. Universidade de Santiago de Compostela. 
Leithwood, K. (2009). Cómo liderar nuestras escuelas: aportes desde la investigación. Santiago, Chile: Fundación Chile.

Martin, E. \& Martínez Rizo, F. (2009). Evaluación: avances y desafíos en la evaluación educativa. Madrid: Organización de estados Iberoamericanos para la educación, la ciencia y la cultura: Fundación Santillana para Iberoamérica.

Meza, M. E. (2008). Reflexiones en torno a la evaluación de la calidad educativa en América Latina y el Caribe. Santiago, Chile: LLECE, OREALC/UNESCO.

Mingorance, P. \& Estebaranz, A. (2009). Construyendo la comunidad que aprende: la vinculación efectiva entre la escuela y la comunidad. Revista Fuentes, (9), 179-199. Retrieved from https://ojs.publius. us.es/ojs/index.php/fuentes/article/view/2546

OECD (Ed.). (2009). Creating effective teaching and learning environments: first results from TALIS. Paris: OECD.

OECD. (2014). TALIS 2013 Results. OECD Publishing. Recuperado a partir de http://www.oecd-ilibrary.org/ education/talis-2013-results_9789264196261-en

Poggi, M. (comp.)(1998) Apuntes y aportes para la gestion curricular. Editorial Kapelusz. Buenos Aires

Rodríguez, G. (2016). Análisis teórico-reflexivo sobre los factores que intervienen en la calidad de los aprendizajes y práctica docente. Revista Gestión Educativa, Vol.6, №, pp.103-119. Escuela de Administración Educativa de la Universidad de Costa Rica. Consultado el 28 de abril de 2017 desde http://dx.doi.org/10.15517/rge.v1i1.22723

Tenti, E. (2004). Notas sobre escuela y comunidad. Buenos Aires. UNESCO. Recuperado en: https://unesdoc.unesco.org/ark:/48223/pf0000141735

UNESCO. (2016). Educación 2030. Declaración de Incheon y Marco de Acción para la realización del objetivo de Desarrollo Sostenible 4. Recuperado a partir de https://unesdoc.unesco.org/ark:/48223/ pf0000245656_spa 